\title{
PRESERVATION OF CONVERGENCE OF CONVEX SETS AND FUNCTIONS IN FINITE DIMENSIONS
}

\author{
BY
}

\author{
L. MCLINDEN AND ROY C. BERGSTROM ${ }^{1}$
}

\begin{abstract}
We study a convergence notion which has particular relevance for convex analysis and lends itself quite naturally to successive approximation schemes in a variety of areas. Motivated particularly by problems in optimization subject to constraints, we develop technical tools necessary for systematic use of this convergence in finite-dimensional settings. Simple conditions are established under which this convergence for sequences of sets, functions and subdifferentials is preserved under various basic operations, including, for example, those of addition and infimal convolution in the case of functions.
\end{abstract}

1. Introduction. A certain very natural notion of convergence for sequences of convex sets, functions and related operators has received increasing attention since the mid-1960's. Such work has been motivated by efforts toward successive approximation schemes in a wide variety of areas, including statistics, variational inequalities, approximation theory, stochastic optimization, control theory, and mathematical programming. Especially noteworthy features of this convergence notion, as applied to convex functions, are that it is preserved under two of the chief operations of convex analysis: the Fenchel transform, and subdifferentiation. In the context of functions, the notion differs in subtle yet important ways from ordinary pointwise convergence.

In this paper we analyze how this convergence behaves with respect to various basic constructions involving convex sets, functions and subdifferentials. Our aim is to develop the technical tools necessary for a systematic application of this convergence notion to constrained optimization situations. Elsewhere [4], [10] we make essential use of the tools developed here. For example, in [4] we develop the details of a broad scheme for approximating a given dual pair of optimization problems as the limit of a sequence of such pairs of problems. Since the source of the sequence of approximating problems is left unspecified, the framework permits applications of both a theoretical and a numerical analytical nature.

The plan of the paper is as follows. In $\$ 2$ we define the convergence notion for sets, functions and operators, and then the two basic results concerning preservation under conjugacy and subdifferentiation are recalled. In $\$ 3$ we treat preservation of convergence of sequences of convex sets under the operations of addition

Received by the editors September 19, 1980 and, in revised form, October 13, 1980.

1980 Mathematics Subject Classification. Primary 90C25; Secondary 26B25, 41A29, 49D45, 65K05.

Key words and phrases. Successive approximation, constrained optimization, convergence, convex analysis, dual operations, subdifferentials, infimal convolution, conjugate duality, separable functions.

${ }^{1}$ Research supported in part by the National Science Foundation under grant number MCS 79-05793 at the University of Illinois at Urbana-Champaign. 
and forming images under linear transformations. Building on these results, we then treat sequences of convex functions with respect to the dual operations of addition and infimal convolution in $\$ 4$ and also two other dual operations involving linear transformations in $\S 6$. The latter two operations are central to applications to more general programming situations [4], [10]. In $\S 5$ we consider the case of additively separable functions. This permits application in decomposition situations, as well as in network optimization [4]. In $\$ 7$ the result concerning addition of functions is recast in terms of the associated subdifferential operators. This gives a sum result for this basic class of maximal monotone operators. Additional such results for other classes of maximal monotone operators can be found in [2], [3]. Finally, in $\$ 8$ we present generalizations of the earlier results which involved two sequences of functions. These extensions from the case $k=2$ to $k>2$ are not necessarily immediate, as one would customarily expect. This is due to the sometimes rather intricate juggling of hypotheses needed to accomodate the duality arguments used.

2. Definitions and background results. First, we recall a well-known notion of convergence for sequences of subsets of $R^{n}$. For each index $\alpha=1,2, \ldots$ let $S_{\alpha}$ be a subset of $R^{n}$. The limit inferior of the sequence $\left(S_{\alpha}\right)$ is the set

$$
\lim \inf S_{\alpha}=\left\{\lim x_{\alpha} \mid x_{\alpha} \in S_{\alpha}, \alpha \in(\alpha)\right\},
$$

and the limit superior of $\left(S_{\alpha}\right)$ is the set

$$
\lim \sup S_{\alpha}=\left\{\lim x_{\beta} \mid x_{\beta} \in S_{\beta}, \beta \in(\beta),(\beta) \subset(\alpha)\right\} .
$$

Here we write $(\alpha)$ to denote the sequence of integers $\alpha=1,2, \ldots$ (excluding the limit index $\infty)$, and we write $(\beta) \subset(\alpha)$ to denote that $(\beta)$ is a subsequence of $(\alpha)$. The sequence $\left(S_{\alpha}\right)$ converges to $S_{\infty}$ if and only if

$$
\lim \sup S_{\alpha} \subset S_{\infty} \subset \lim \inf S_{\alpha}
$$

which we denote by writing $S_{\alpha} \rightarrow S_{\infty}$. If $\left(S_{\alpha}\right)$ converges to $S_{\infty}$, then we actually have equality throughout (2.1) and $S_{\infty}$ is closed.

If $f$ is an extended-real-valued function on $R^{n}$, its epigraph is the set

$$
\text { epi } f=\left\{(x, \mu) \in R^{n+1} \mid \mu \geqslant f(x)\right\} \text {. }
$$

For each $\alpha=1, \ldots, \infty$ let $f_{\alpha}$ be such a function. We say the sequence $\left(f_{\alpha}\right)$ converges to $f_{\infty}$, written $f_{\alpha} \rightarrow f_{\infty}$, if and only if the sequence (epi $f_{\alpha}$ ) of epigraphs converges to epi $f_{\infty}$ in the sense of (2.1).

This convergence of functions is in general not comparable to pointwise convergence, even in the case in which each $f_{\alpha}$ is closed proper convex, i.e. when each epi $f_{\alpha}$ is a nonempty closed convex set containing no vertical line. Elementary examples illustrating the inequivalence can be found in [18] and [20]. For an extensive analysis of the relationship between the present convergence and others, see Salinetti and Wets [15], [16]. In particular, in [15] a maximal class of closed proper convex functions is identified for which pointwise convergence and the present notion coincide.

The special importance which the convergence defined above has for convex functions is foreshadowed by the following two theorems. For these, a few 
definitions will now be recalled. (Further background on convex analysis can be found in Rockafellar [13].) The effective domain of a convex function is the set

$$
\operatorname{dom} f=\left\{x \in R^{n} \mid f(x)<+\infty\right\} \text {. }
$$

The conjugate (or Fenchel transform) of $f$ is the closed convex function $f^{*}$ given by

$$
f^{*}\left(x^{*}\right)=\sup _{x}\left\{x \cdot x^{*}-f(x)\right\}, \quad x^{*} \in R^{n},
$$

where we have used the notation $x \cdot x^{*}$ to denote the usual inner product of two vectors.

THEOREM 1. For each $\alpha=1, \ldots, \infty$ let $f_{\alpha}$ be a closed proper convex function on $R^{n}$. Then $f_{\alpha} \rightarrow f_{\infty}$ if and only if $f_{\alpha}^{*} \rightarrow f_{\infty}^{*}$.

This result was proved by Wijsman [20]. Independently, Walkup and Wets [17] established for reflexive Banach spaces a closely related result involving polar cones. Their result, incorporating a metric viewpoint, is in a sense stronger and, as was pointed out by R. T. Rockafellar, implies Theorem 1 via [13, Theorem 14.4]. Banach space extensions of Theorem 1 per se have been established by Mosco [12] and Joly [6], [7]. A nonconvex version of Theorem 1 is given in McLinden [9].

A second main background result relates convergence of convex functions to that of the associated sequence of subdifferentials. Recall that the set of subgradients of $f$ at $x$ is the set

$$
\partial f(x)=\left\{x^{*} \in R^{n} \mid f\left(x^{\prime}\right) \geqslant f(x)+\left(x^{\prime}-x\right) \cdot x^{*}, \forall x^{\prime} \in R^{n}\right\} .
$$

The subdifferential of $f$ is the multivalued operator $\partial f$ mapping points $x \in R^{n}$ into subsets $\partial f(x) \subset R^{n}$ and having graph

$$
G(\partial f)=\left\{\left(x, x^{*}\right) \in R^{2 n} \mid x^{*} \in \partial f(x)\right\} .
$$

For convenience in what follows, we identify subdifferentials with their graphs. The effective domain of $\partial f$ is the set

$$
D(\partial f)=\left\{x \in R^{n} \mid \partial f(x) \neq \varnothing\right\} .
$$

THEOREM 2. For each $\alpha=1, \ldots, \infty$ let $f_{\alpha}$ be a closed proper convex function on $R^{n}$. Then $f_{\alpha} \rightarrow f_{\infty}$ if and only if

(i) $G\left(\partial f_{\alpha}\right) \rightarrow G\left(\partial f_{\infty}\right)$ and

(ii) there exist $\left(x_{\alpha}, x_{\alpha}^{*}\right) \in G\left(\partial f_{\alpha}\right)$ for $\alpha=1, \ldots, \infty$ such that $\left(x_{\infty}, x_{\infty}^{*}\right)=$ $\lim \left(x_{\alpha}, x_{\alpha}^{*}\right)$ and $f_{\infty}\left(x_{\infty}\right)=\lim f_{\alpha}\left(x_{\alpha}\right)$.

This result is due to Attouch [1], who established it in the setting of Hilbert spaces. Related material, variants and alternate proofs can be found in Brézis [5], Matzeu [8], and McLinden [9].

To conclude this preliminary section, we record several more definitions. For any operator $T$ mapping points of $R^{n}$ into subsets (possibly empty or singletons) of $R^{m}$, we write

$$
D(T)=\left\{x \in R^{n} \mid T(x) \neq \varnothing\right\}, \quad G(T)=\left\{(x, y) \in R^{n} \times R^{m} \mid y \in T(x)\right\}
$$

for the effective domain and the graph of $T$. If $T$ is such an operator for each 
$\alpha=1, \ldots, \infty$, we say that the sequence $\left(T_{\alpha}\right)$ converges to $T_{\infty}$, and write $T_{\alpha} \rightarrow T_{\infty}$, provided that the sequence $\left(G\left(T_{\alpha}\right)\right)$ of graphs converges to $G\left(T_{\infty}\right)$ in the sense of (2.1).

3. Operations on sets. If $A$ is a linear transformation, we denote its nullspace, range, and graph by $N(A), R(A)$, and $G(A)$, respectively. If $C$ is a nonempty convex subset of $R^{n}$, its recession cone is the nonempty convex set

$$
0^{+} C=\left\{y \in R^{n} \mid y+C \subset C\right\} \text {. }
$$

The following result is central to this paper. Notice that the technical condition it requires involves only the limit linear transformation and convex set.

THEOREM 3. For each $\alpha=1, \ldots, \infty$ let $C_{\alpha}$ be a nonempty convex subset of $R^{n}$ and $A_{\alpha}$ be a linear transformation from $R^{n}$ to $R^{m}$. Assume that $C_{\alpha} \rightarrow C_{\infty}$ and $A_{\alpha} \rightarrow A_{\infty}$ (pointwise). Suppose that $N\left(A_{\infty}\right) \cap 0^{+} C_{\infty}=\{0\}$. Then $A_{\alpha} C_{\alpha} \rightarrow A_{\infty} C_{\infty}$. If for each $\alpha$ the set $C_{\alpha}$ is also closed, then there is an $\bar{\alpha}$ such that $\alpha>\bar{\alpha}$ implies $A_{\alpha} C_{\alpha}$ is closed.

Before proving Theorem 3, we draw the following corollary.

Corollary 3A. For each $\alpha=1, \ldots, \infty$ let $C_{\alpha}$ and $D_{\alpha}$ be nonempty convex subsets of $R^{n}$ such that $C_{\alpha} \rightarrow C_{\infty}$ and $D_{\alpha} \rightarrow D_{\infty}$. Suppose that $\left(0^{+} C_{\infty}\right) \cap\left(-0^{+} D_{\infty}\right)$ $=\{0\}$. Then $\left(C_{\alpha}+D_{\alpha}\right) \rightarrow\left(C_{\infty}+D_{\infty}\right)$. If for each $\alpha$ both $C_{\alpha}$ and $D_{\alpha}$ are also closed, then there is an $\bar{\alpha}$ such that $\alpha>\bar{\alpha}$ implies $\left(C_{\alpha}+D_{\alpha}\right)$ is closed.

Proof. For each $\alpha=1, \ldots, \infty$ define $A_{\alpha}: R^{2 n} \rightarrow R^{n}$ by $A_{\alpha}\left(x_{1}, x_{2}\right)=x_{1}+x_{2}$. It is easy to check that the sequence of direct sums $\left(\left(C_{\alpha} \oplus D_{\alpha}\right)\right)$ converges to $C_{\infty} \oplus D_{\infty}$. Since $A_{\alpha}\left(C_{\alpha} \oplus D_{\alpha}\right)=C_{\alpha}+D_{\alpha}$ and $0^{+}\left(C_{\alpha} \oplus D_{\alpha}\right)=0^{+} C_{\alpha} \oplus 0^{+} D_{\alpha}$, the hypothesis $\left(0^{+} C_{\infty}\right) \cap\left(-0^{+} D_{\infty}\right)=\{0\}$ implies $N\left(A_{\infty}\right) \cap 0^{+}\left(C_{\infty} \oplus D_{\infty}\right)=\{0\}$. The corollary now follows from Theorem 3 .

The proof of Theorem 3 depends on a series of lemmas.

LEMma 1. Suppose that $\left(x_{\alpha}\right)$ and $\left(c_{\alpha}\right)$ are sequences in $R^{n}$ with $\left\|c_{\alpha}\right\| \rightarrow \infty$. Assume there exist $\kappa>0$ and $u \in R^{n}$ such that $\left\|x_{\alpha}\right\|<\kappa$ for all $\alpha$ and $c_{\alpha}\left\|c_{\alpha}\right\|^{-1} \rightarrow u$. Then $\left(c_{\alpha}-x_{\alpha}\right)\left\|c_{\alpha}-x_{\alpha}\right\|^{-1} \rightarrow u$.

Proof. We have $\left\|x_{\alpha}\right\| \leqslant \kappa<\left\|c_{\alpha}\right\|$ for all sufficiently large $\alpha$, and hence

$$
\left(1+\left\|x_{\alpha}\right\|\left\|c_{\alpha}\right\|^{-1}\right)^{-1} \leqslant\left\|c_{\alpha}\right\|\left\|c_{\alpha}-x_{\alpha}\right\|^{-1} \leqslant\left(1-\left\|x_{\alpha}\right\|\left\|c_{\alpha}\right\|^{-1}\right)^{-1}
$$

by the triangle inequality. Thus $\left\|c_{\alpha}\right\|\left\|c_{\alpha}-x_{\alpha}\right\|^{-1} \rightarrow 1$, and consequently

$$
\left(c_{\alpha}-x_{\alpha}\right)\left\|c_{\alpha}-x_{\alpha}\right\|^{-1}=\left(c_{\alpha}\left\|c_{\alpha}\right\|^{-1}-x_{\alpha}\left\|c_{\alpha}\right\|^{-1}\right)\left\|_{\alpha}\right\|\left\|c_{\alpha}-x_{\alpha}\right\|^{-1} \rightarrow u,
$$

completing the proof.

LemMa 2. For each $\alpha=1, \ldots, \infty$ let $C_{\alpha}$ be a nonempty convex subset of $R^{n}$. Assume that $C_{\alpha} \rightarrow C_{\infty}$. Suppose for some subsequence $\left(C_{\beta}\right)$ of $\left(C_{\alpha}\right)$ and $u \in R^{n}$ there is a sequence $\left(c_{\beta}\right)$ such that $c_{\beta} \in C_{\beta},\left\|c_{\beta}\right\| \rightarrow \infty$, and $c_{\beta}\left\|c_{\beta}\right\|^{-1} \rightarrow u$. Then $u \in$ $0^{+} C_{\infty}$. 
Proof. Choose $x \in C_{\infty}$ and $r>0$. It will suffice to show $(x+r u) \in C_{\infty}$. We can find a sequence $\left(x_{\alpha}\right)$ such that $x_{\alpha} \in C_{\alpha}$ and $x_{\alpha} \rightarrow x$, and then we can choose $\kappa>0$ such that $\left\|x_{\alpha}\right\|<\kappa$ for all $\alpha$. Let $\left(x_{\beta}\right)$ be the subsequence of $\left(x_{\alpha}\right)$ corresponding to the subsequence $\left(C_{\beta}\right)$ of the hypothesis. There exists $\bar{\beta}$ such that $\beta>\bar{\beta}$ implies $\left\|c_{\beta}\right\|>(\kappa+r)$. For such $\beta$ we have that $0<r<\left\|c_{\beta}-x_{\beta}\right\|$ and (since $C_{\beta}$ is convex)

$$
x_{\beta}+\lambda\left\|c_{\beta}-x_{\beta}\right\|\left(\frac{c_{\beta}-x_{\beta}}{\left\|c_{\beta}-x_{\beta}\right\|}\right) \in C_{\beta} \quad \text { for } 0 \leqslant \lambda \leqslant 1 \text {, }
$$

so that

$$
x_{\beta}+r\left(\left(c_{\beta}-x_{\beta}\right) /\left\|c_{\beta}-x_{\beta}\right\|\right) \in C_{\beta} .
$$

It follows from Lemma 1 that $\left(c_{\beta}-x_{\beta}\right)\left\|c_{\beta}-x_{\beta}\right\|^{-1} \rightarrow u$, and this combined with $x_{\beta} \rightarrow x$ and $C_{\alpha} \rightarrow C_{\infty}$ yields $(x+r u) \in C_{\infty}$.

LEMMA 3. For each $\alpha=1, \ldots, \infty$ let $A_{\alpha}$ be a linear transformation from $R^{n}$ to $R^{m}$. Then the following conditions are pairwise equivalent:

$$
\begin{gathered}
G\left(A_{\alpha}\right) \rightarrow G\left(A_{\infty}\right), \\
A_{\alpha} \rightarrow A_{\infty} \quad \text { pointwise }, \\
\left\|A_{\alpha}-A_{\infty}\right\| \rightarrow 0 .
\end{gathered}
$$

(The convergence in (3.1) is as described in $\$ 2$, where the graphs are considered as subsets of $R^{n} \times R^{m}$.)

Proof. First, we show (3.1) implies (3.2). We begin by noticing that $\left(\left\|A_{\alpha}\right\|\right)$ is bounded. Indeed, suppose otherwise. Then there is a subsequence $\left(A_{\beta}\right)$ of $\left(A_{\alpha}\right)$ such that $\left\|A_{\beta}\right\| \rightarrow \infty$. Choose a sequence $\left(u_{\beta}\right)$ of unit vectors such that $\left\|A_{\beta} u_{\beta}\right\|>$ $\left(\left\|A_{\beta}\right\| / 2\right)$, and set $\gamma_{\beta}=\left\|A_{\beta} u_{\beta}\right\|^{-1}$. Since $\left\|A_{\beta}\left(\gamma_{\beta} u_{\beta}\right)\right\|=1$ for all $\beta$, we may assume that there is a unit vector $v \in R^{m}$ such that $A_{\beta}\left(\gamma_{\beta} u_{\beta}\right) \rightarrow v$. From the hypothesis $G\left(A_{\alpha}\right) \rightarrow G\left(A_{\infty}\right)$ and $\left(\gamma_{\beta} u_{\beta}, A_{\beta}\left(\gamma_{\beta} u_{\beta}\right)\right) \rightarrow(0, v)$ we conclude that $A_{\infty}(0)=v$. But this is a contradiction, since $v$ is a unit vector. Now let $x_{\infty} \in R^{n}$. Since $G\left(A_{\alpha}\right) \rightarrow$ $G\left(A_{\infty}\right)$, there is a sequence $\left(x_{\alpha}\right)$ in $R^{n}$ such that $\left(x_{\alpha}, A_{\alpha} x_{\alpha}\right) \rightarrow\left(x_{\infty}, A_{\infty} x_{\infty}\right)$. In view of the estimate

$$
\left\|A_{\infty} x_{\infty}-A_{\alpha} x_{\infty}\right\| \leqslant\left\|A_{\infty} x_{\infty}-A_{\alpha} x_{\alpha}\right\|+\left\|A_{\alpha}\right\|\left\|x_{\alpha}-x_{\infty}\right\|
$$

the desired implication follows. That (3.2) is equivalent to (3.3) is standard. Finally, we show (3.2) and (3.3) imply (3.1). We have $G\left(A_{\infty}\right) \subset \lim \inf G\left(A_{\alpha}\right)$ since $\left(x_{\infty}, A_{\alpha} x_{\infty}\right) \rightarrow\left(x_{\infty}, A_{\infty} x_{\infty}\right)$. Suppose $\left(G\left(A_{\beta}\right)\right)$ is a subsequence of $\left(G\left(A_{\alpha}\right)\right)$ and $\left(x_{\beta}\right)$ is a sequence such that the sequence $\left(\left(x_{\beta}, A_{\beta} x_{\beta}\right)\right)$ converges to some pair $\left(x_{\infty}, y\right)$. To prove lim sup $G\left(A_{\alpha}\right) \subset G\left(A_{\infty}\right)$ it will suffice to show $y=A_{\infty} x_{\infty}$. But this is immediate, since

$$
\left\|y-A_{\infty} x_{\infty}\right\| \leqslant\left\|y-A_{\beta} x_{\beta}\right\|+\left\|A_{\beta}\right\|\left\|x_{\beta}-x_{\infty}\right\|+\left\|A_{\beta}-A_{\infty}\right\|\left\|x_{\infty}\right\|,
$$

and the quantity on the right converges to zero.

In view of Lemma 3 , henceforth we write $A_{\alpha} \rightarrow A_{\infty}$ for any of these three equivalent forms of convergence of linear transformations. 
LemMA 4. For each $\alpha=1, \ldots, \infty$ let $C_{\alpha}$ be a nonempty convex subset of $R^{n}$. Assume that $C_{\alpha} \rightarrow C_{\infty}$. Then $\lim \sup 0^{+} C_{\alpha} \subset 0^{+} C_{\infty}$.

Proof. Suppose that $y_{\infty} \in \lim \sup \left(0^{+} C_{\alpha}\right)$. Then we can find a subsequence $\left(0^{+} C_{\beta}\right)$ of $\left(0^{+} C_{\alpha}\right)$ and a sequence $\left(y_{\beta}\right)$ such that $y_{\beta} \in 0^{+} C_{\beta}$ and $y_{\beta} \rightarrow y_{\infty}$. Choose $x_{\infty} \in C_{\infty}$ and $r>0$. It will suffice to show that $\left(x_{\infty}+r y_{\infty}\right) \in C_{\infty}$. Since $C_{\alpha} \rightarrow$ $C_{\infty}$, there is a sequence $\left(x_{\alpha}\right)$ such that $x_{\alpha} \in C_{\alpha}$ and $x_{\alpha} \rightarrow x_{\infty}$. Now $\left(x_{\beta}+r y_{\beta}\right) \in C_{\beta}$ and $\lim \sup C_{\beta} \subset C_{\infty}$, so $\left(x_{\infty}+r y_{\infty}\right) \in C_{\infty}$.

Lemma 5. For each $\alpha=1, \ldots, \infty$ let $C_{\alpha}$ and $D_{\alpha}$ be convex cones in $R^{n}$. Assume that $\lim \sup C_{\alpha} \subset C_{\infty}$ and $\lim \sup D_{\alpha} \subset D_{\infty}$. Suppose that $C_{\infty} \cap D_{\infty}=\{0\}$. Then there is an $\bar{\alpha}$ such that $\alpha>\bar{\alpha}$ implies $C_{\alpha} \cap D_{\alpha}=\{0\}$.

Proof. Suppose the conclusion fails. Then we can find a subsequence $(\beta)$ of $(\alpha)$ and a sequence of nonzero vectors $\left(u_{\beta}\right)$ such that $u_{\beta} \in C_{\beta} \cap D_{\beta}$. We may assume that each $u_{\beta}$ is a unit vector and that there is a unit vector $u$ such that $u_{\beta} \rightarrow u$. Then $u \in C_{\infty} \cap D_{\infty}$ by the "lim sup" hypothesis. This contradiction shows the conclusion must be valid.

Proof of Theorem 3. The set $A_{\alpha} C_{\alpha}$ is clearly nonempty convex. Let $c_{\infty} \in C_{\infty}$. Since $C_{\infty} \subset \lim \inf C_{\alpha}$, there is a sequence $\left(c_{\alpha}\right)$ with $c_{\alpha} \in C_{\alpha}$ such that $c_{\alpha} \rightarrow c_{\infty}$. It follows that $A_{\infty} c_{\infty} \in \lim \inf A_{\alpha} C_{\alpha}$, since

$$
\left\|A_{\alpha} c_{\alpha}-A_{\infty} c_{\infty}\right\| \leqslant\left\|A_{\alpha}-A_{\infty}\right\|\left\|c_{\alpha}\right\|+\left\|A_{\infty}\right\|\left\|c_{\alpha}-c_{\infty}\right\|
$$

and the quantity on the right goes to zero as $\alpha$ goes to infinity. Now let $\left(C_{\beta}\right)$ be a subsequence of $\left(C_{\alpha}\right)$ and $\left(c_{\beta}\right)$ be a sequence such that $c_{\beta} \in C_{\beta}$ and the sequence $A_{\beta} c_{\beta}$ converges to some $x_{\infty} \in R^{m}$. To complete the convergence conclusion it will suffice to show that $x_{\infty}=A_{\infty} c_{\infty}$ for some $c_{\infty} \in C_{\infty}$. We claim that the sequence $\left(\left\|c_{\beta}\right\|\right)$ is bounded. If not, we may assume that $\left\|c_{\beta}\right\| \rightarrow \infty$ and that there is a unit vector $u \in R^{n}$ such that $c_{\beta}\left\|c_{\beta}\right\|^{-1} \rightarrow u$. From Lemma 2 it follows that $u \in 0^{+} C_{\infty}$. Let $u_{\beta}=c_{\beta}\left\|c_{\beta}\right\|^{-1}$. Since $A_{\beta} \rightarrow A_{\infty}$ pointwise and $u_{\beta} \rightarrow u$, we have $A_{\beta} u_{\beta} \rightarrow A_{\infty} u$. We also have $\left\|c_{\beta}\right\|\left(A_{\beta} u_{\beta}\right) \rightarrow x_{\infty}$ and so $\left\|c_{\beta}\right\|\left\|A_{\beta} u_{\beta}\right\| \rightarrow\left\|x_{\infty}\right\|$. This implies $\left(\left\|A_{\beta} u_{\beta}\right\|\right) \rightarrow 0$, since $\left\|c_{\beta}\right\| \rightarrow \infty$, and so $u \in N\left(A_{\infty}\right)$. But this contradicts the hypothesis $N\left(A_{\infty}\right) \cap 0^{+} C_{\infty}=\{0\}$. Therefore $\left(\left\|c_{\beta}\right\|\right)$ is bounded, and we may assume $\left(c_{\beta}\right)$ converges to some vector $c_{\infty}$. Since $\lim \sup C_{\alpha} \subset C_{\infty}$, it follows that $c_{\infty} \in C_{\infty}$. The estimate

$$
\left\|A_{\beta} c_{\beta}-A_{\infty} c_{\infty}\right\| \leqslant\left\|A_{\beta}\right\|\left\|c_{\beta}-c_{\infty}\right\|+\left\|A_{\beta}-A_{\infty}\right\|\left\|c_{\infty}\right\|,
$$

together with $A_{\beta} c_{\beta} \rightarrow x_{\infty}$ and $A_{\beta} \rightarrow A_{\infty}$, implies that $x_{\infty}=A_{\infty} c_{\infty}$.

Finally, suppose that for each $\alpha$ the set $C_{\alpha}$ is also closed. Lemma 4 implies lim sup $0^{+} C_{\alpha} \subset 0^{+} C_{\infty}$, and $A_{\alpha} \rightarrow A_{\infty}$ implies lim sup $N\left(A_{\alpha}\right) \subset N\left(A_{\infty}\right)$. Lemma 5 then implies that there is an $\bar{\alpha}$ such that $\alpha>\bar{\alpha}$ implies $N\left(A_{\alpha}\right) \cap 0^{+} C_{\alpha}=\{0\}$. It follows from [13, Theorem 9.1] that for these $\alpha$ the set $A_{\alpha} C_{\alpha}$ is closed.

In Theorem 3 the hypothesis $N\left(A_{\infty}\right) \cap 0^{+} C_{\infty}=\{0\}$ cannot in general be relaxed to " $N\left(A_{\infty}\right) \cap 0^{+} C_{\infty}$ is a subspace."

EXAMPLE 1. For each $\alpha=1, \ldots, \infty$ define $A_{\alpha}: R^{2} \rightarrow R^{2}$ by $A_{\alpha}(x, y)=(0, y)$. For $\alpha<\infty$ let $C_{\alpha}$ be the triangle in $R^{2}$ with vertices $(-\alpha, 0),(\alpha, 0)$, and $(\alpha, 1)$, and let $C_{\infty}=R \times\{0\}$. Then $A_{\infty} C_{\infty}=\{(0,0)\}$, and for $\alpha<\infty, A_{\alpha} C_{\alpha}=\{0\} \times[0,1]$. 
Here $C_{\alpha} \rightarrow C_{\infty}$ and $N\left(A_{\infty}\right) \cap 0^{+} C_{\infty}$ is the simplest nontrivial subspace, yet $\left(A_{\alpha} C_{\alpha}\right)$ does not converge to $A_{\infty} C_{\infty}$.

4. Addition and inf-convolution of functions. We now apply the results of $\$ 3$ to epigraphs of functions to obtain results for preservation of convergence of functions under natural operations. The recession function of a closed proper convex function $f$ on $R^{n}$ is the function whose epigraph is $0^{+}$(epi $f$ ). It is denoted by $f 0^{+}$. The infimal convolution of two closed proper convex functions $f$ and $g$ on $R^{n}$ is the convex function on $R^{n}$ given by

$$
(f \square g)(x)=\inf _{x^{\prime}}\left\{f\left(x^{\prime}\right)+g\left(x-x^{\prime}\right)\right\} .
$$

THEOREM 4. For each $\alpha=1, \ldots, \infty$ let $f_{\alpha}$ and $g_{\alpha}$ be closed proper convex functions on $R^{n}$. Assume that $f_{\alpha} \rightarrow f_{\infty}$ and $g_{\alpha} \rightarrow g_{\infty}$. Suppose that $0^{+}\left(\right.$epi $\left.f_{\infty}\right) \cap$ $\left(-0^{+}\left(\right.\right.$epi $\left.\left.g_{\infty}\right)\right)=\{0\}$. Then there is an $\bar{\alpha}$ such that $\alpha>\bar{\alpha}$ implies $\left(f_{\alpha} \square g_{\alpha}\right)$ is closed proper convex, and $\left(f_{\alpha} \square g_{\alpha}\right) \rightarrow\left(f_{\infty} \square g_{\infty}\right)$.

Proof. The definition of infimal convolution implies that for all $\alpha$,

$$
\text { (epi } \left.f_{\alpha}+\text { epi } g_{\alpha}\right) \subset \operatorname{epi}\left(f_{\alpha} \square g_{\alpha}\right) \subset \operatorname{cl}\left(\text { epi } f_{\alpha}+\text { epi } g_{\alpha}\right) \text {. }
$$

Corollary $3 \mathrm{~A}$ implies that there is an $\bar{\alpha}$ such that $\alpha>\bar{\alpha}$ implies (epi $f_{\alpha}+$ epi $g_{\alpha}$ ) is closed, and that the sequence ((epi $f_{\alpha}+$ epi $\left.g_{\alpha}\right)$ ) converges to (epi $f_{\infty}+$ epi $\left.g_{\infty}\right)$. By Lemma 5, we may assume that $\alpha>\bar{\alpha}$ implies $0^{+}\left(\right.$epi $\left.f_{\alpha}\right) \cap\left(-0^{+}\left(\right.\right.$epi $\left.\left.g_{\alpha}\right)\right)=\{0\}$. For these $\alpha$ we have from [13, Corollary 9.2.2] (see also Lemma 6 below) that $f_{\alpha} \square g_{\alpha}$ is closed proper convex.

We now investigate dual formulations of the hypothesis of Theorem 4, in preparation for Theorem 5 .

LEMMA 6. Let $f$ and $g$ be closed proper convex functions on $R^{n}$. Then the following three conditions

$$
\begin{gathered}
0^{+}\left(\text {epi } f^{*}\right) \cap\left(-0^{+}\left(\text {epi } g^{*}\right)\right)=\{0\} \\
\left(f^{*} 0^{+}\right)\left(x^{*}\right)+\left(g^{*} 0^{+}\right)\left(-x^{*}\right)>0 \text { for all } x^{*} \neq 0, \\
0 \in \operatorname{int}(\operatorname{dom} f-\operatorname{dom} g),
\end{gathered}
$$

are pairwise equivalent, and are implied by

$$
\varnothing \neq \operatorname{int}(\operatorname{dom} f) \cap \operatorname{dom} g .
$$

Proof. Condition (4.3) is equivalent to having $R^{n}$ equal to the convex cone generated by the effective domain of the indicator function $\delta(\cdot \mid \operatorname{dom} f-\operatorname{dom} g)$. It follows from [13, Theorem 14.2] that this is equivalent to having the recession cone of the support function $\delta^{*}(\cdot \mid \operatorname{dom} f-\operatorname{dom} g)$ consist of just the zero vector. Notice that

$$
\begin{aligned}
\delta^{*}\left(x^{*} \mid \operatorname{dom} f-\operatorname{dom} g\right) & =\delta^{*}\left(x^{*} \mid \operatorname{dom} f\right)+\delta^{*}\left(x^{*} \mid-\operatorname{dom} g\right) \\
& =\left(f^{*} 0^{+}\right)\left(x^{*}\right)+\left(g^{*} 0^{+}\right)\left(-x^{*}\right),
\end{aligned}
$$


where the second equality follows from [13, Theorem 13.3]. We conclude that (4.2) is equivalent to (4.3). Now suppose that

$$
\left(x^{*}, \mu^{*}\right) \in 0^{+}\left(\text {epi } f^{*}\right) \cap\left(-0^{+}\left(\text {epi } g^{*}\right)\right) \text {. }
$$

Then we have $\mu^{*} \geqslant\left(f^{*} 0^{+}\right)\left(x^{*}\right)$ and $-\mu^{*} \geqslant\left(g^{*} 0^{+}\right)\left(-x^{*}\right)$. In particular, this yields

$$
\left(f^{*} 0^{+}\right)\left(x^{*}\right)+\left(g^{*} 0^{+}\right)\left(-x^{*}\right) \leqslant \mu^{*}-\mu^{*}=0
$$

so that (4.2) implies $x^{*}=0$. It follows that

$$
\mu^{*} \geqslant\left(f^{*} 0^{+}\right)\left(x^{*}\right)=0 \text { and }-\mu^{*} \geqslant\left(g^{*} 0^{+}\right)\left(-x^{*}\right)=0,
$$

which imply $\mu^{*}=0$. Thus (4.2) implies (4.1). We establish the implication $(4.1) \Rightarrow$ (4.3) by contraposition. Suppose that there exists $x^{*} \neq 0$ such that $\left(f^{*} 0^{+}\right)\left(x^{*}\right)+$ $\left(g^{*} 0^{+}\right)\left(-x^{*}\right) \leqslant 0$. Then both summands are finite (see [13, Theorem 8.5]), so there exist real numbers $\mu^{*}$ and $\nu^{*}$ satisfying $\left(f^{*} 0^{+}\right)\left(x^{*}\right) \leqslant \mu^{*},\left(g^{*} 0^{+}\right)\left(-x^{*}\right) \leqslant-\nu^{*}$, and $\mu^{*}-\nu^{*} \leqslant 0$. These conditions yield

$$
\left(x^{*}, \mu^{*}\right) \in 0^{+}\left(\text {epi } f^{*}\right) \cap\left(-0^{+}\left(\text {epi } g^{*}\right)\right) .
$$

Finally, (4.4) $\Rightarrow(4.3)$ follows from

$$
0 \in(\operatorname{int}(\operatorname{dom} f)-\operatorname{dom} g) \subset \operatorname{int}(\operatorname{dom} f-\operatorname{dom} g),
$$

and the proof is complete.

THEOREM 5. For each $\alpha=1, \ldots, \infty$ let $f_{\alpha}$ and $g_{\alpha}$ be closed proper convex functions on $R^{n}$. Assume that $f_{\alpha} \rightarrow f_{\infty}$ and $g_{\alpha} \rightarrow g_{\infty}$. Suppose that

$$
0 \in \operatorname{int}\left(\operatorname{dom} f_{\infty}-\operatorname{dom} g_{\infty}\right) \text {. }
$$

Then there is an $\bar{\alpha}$ such that $\alpha>\bar{\alpha}$ implies $\left(f_{\alpha}+g_{\alpha}\right)$ is closed proper convex, and $\left(f_{\alpha}+g_{\alpha}\right) \rightarrow\left(f_{\infty}+g_{\infty}\right)$.

Proof. It follows from Theorem 1 that $f_{\alpha}^{*} \rightarrow f_{\infty}^{*}$ and $g_{\alpha}^{*} \rightarrow g_{\infty}^{*}$, and it follows from Lemma 6 that $0^{+}\left(\right.$epi $\left.f_{\infty}^{*}\right) \cap\left(-0^{+}\left(\right.\right.$epi $\left.\left.g_{\infty}^{*}\right)\right)=\{0\}$. Hence, Theorem 4 yields an $\bar{\alpha}$ such that $\alpha>\bar{\alpha}$ implies $\left(f_{\alpha}^{*} \square g_{\alpha}^{*}\right)$ is closed proper convex, and $\left(f_{\alpha}^{*} \square g_{\alpha}^{*}\right) \rightarrow$ $\left(f_{\infty}^{*} \square g_{\infty}^{*}\right)$. It follows from [13, Theorems 16.4, 12.2] that for these $\alpha,\left(f_{\alpha}^{*} \square g_{\alpha}^{*}\right)=$ $\left(f_{\alpha}+g_{\alpha}\right)^{*}$ and $\left(f_{\alpha}+g_{\alpha}\right)$ is closed proper convex. The result now follows from another application of Theorem 1.

The relative interior of a subset $S$ of $R^{n}$, denoted by ri $S$, is the interior of $S$ relative to the smallest affine subset of $R^{n}$ which contains $S$. The next example shows that we cannot weaken the technical condition of Theorem 5 from $0 \in$ $\operatorname{int}\left(\operatorname{dom} f_{\infty}-\operatorname{dom} g_{\infty}\right)$ to $0 \in \operatorname{ri}\left(\operatorname{dom} f_{\infty}-\operatorname{dom} g_{\infty}\right)$.

EXAMPLE 2. Define closed proper convex functions on $R^{2}$ by

$f_{\alpha}=\delta(\cdot \mid[0,2] \times\{1 / \alpha\})$ for $\alpha<\infty$,

$f_{\infty}=\delta(\cdot \mid[0,2] \times\{0\})$,

$g_{\alpha}=\delta(\cdot \mid[1,3] \times\{-1 / \alpha\})$ for $\alpha<\infty$,

$g_{\infty}=\delta(\cdot \mid[1,3] \times\{0\})$.

These are all closed proper convex functions, and $f_{\alpha} \rightarrow f_{\infty}$ and $g_{\alpha} \rightarrow g_{\infty}$. The sequence $\left(\left(f_{\alpha}+g_{\alpha}\right)\right)$ does not converge to $\left(f_{\infty}+g_{\infty}\right)$, even though $0 \in$ $\operatorname{ri}\left(\operatorname{dom} f_{\infty}-\operatorname{dom} g_{\infty}\right)$. 
5. Separable functions. Functions which are additively separable over various components of the argument play an important role in applications. Decomposition principles and network optimization constitute outstanding examples of this. The completely separable case is, of course, included in the following result.

THEOREM 6. For each $\alpha=1, \ldots, \infty$ and $i=1, \ldots, k$ let $f_{\alpha}^{i}$ be a closed proper convex function on $R^{m_{i}}$. Assume that $f_{\alpha}^{i} \rightarrow f_{\infty}^{i}$ for each $i$. For $n=m_{1}+\cdots+m_{k}$ define functions $F_{\alpha}$ on $R^{n}$ by setting

$$
F_{\alpha}(x)=f_{\alpha}^{1}\left(x^{1}\right)+\cdots+f_{\alpha}^{k}\left(x^{k}\right), \quad x=\left(x^{1}, \ldots, x^{k}\right) .
$$

Then $F_{\alpha} \rightarrow F_{\infty}$.

Proof. A completely elementary proof can be based on the lemma of Mosco [11, Lemma 1.10] which converts (2.1) into equivalent statements involving function values. Suppose $\left(x_{\infty}^{1}, \ldots, x_{\infty}^{k}\right)$ is given. Since epi $f_{\infty}^{i} \subset \lim \inf$ epi $f_{\alpha}^{i}$ for each $i$, there exists sequences $\left(x_{\alpha}^{i}\right)$ such that

$$
x_{\infty}^{i}=\lim x_{\alpha}^{i} \text { and } \lim \sup f_{\alpha}^{i}\left(x_{\alpha}^{i}\right) \leqslant f_{\infty}^{i}\left(x_{\infty}^{i}\right) .
$$

But then $\left(x_{\infty}^{1}, \ldots, x_{\infty}^{k}\right)=\lim \left(x_{\alpha}^{1}, \ldots, x_{\alpha}^{k}\right)$ and

$$
\begin{aligned}
\lim \sup F_{\alpha}\left(x_{\alpha}^{1}, \ldots, x_{\alpha}^{k}\right) & =\lim \sup \sum f_{\alpha}^{i}\left(x_{\alpha}^{i}\right) \leqslant \sum \lim \sup f_{\alpha}^{i}\left(x_{\alpha}^{i}\right) \\
& \leqslant \sum f_{\infty}^{i}\left(x_{\infty}^{i}\right)=F_{\infty}\left(x_{\infty}^{1}, \ldots, x_{\infty}^{k}\right),
\end{aligned}
$$

which shows that epi $F_{\infty} \subset \lim \inf$ epi $F_{\alpha}$. Now consider any $(\beta) \subset(\alpha)$ and corresponding $\left(x_{\infty}^{1}, \ldots, x_{\infty}^{k}\right)=\lim \left(x_{\beta}^{1}, \ldots, x_{\beta}^{k}\right)$. Since lim sup epi $f_{\alpha}^{i} \subset$ epi $f_{\infty}^{i}$ for each $i$, we have

$$
f_{\infty}^{i}\left(x_{\infty}^{i}\right) \leqslant \lim \inf f_{\beta}^{i}\left(x_{\beta}^{i}\right) .
$$

Adding these yields

$$
\begin{aligned}
F_{\infty}\left(x_{\infty}^{1}, \ldots, x_{\infty}^{k}\right) & =\sum f_{\infty}^{i}\left(x_{\infty}^{i}\right) \leqslant \sum \lim \inf f_{\beta}^{i}\left(x_{\beta}^{i}\right) \\
& \leqslant \liminf \sum f_{\beta}^{i}\left(x_{\beta}^{i}\right)=\lim \inf F_{\beta}\left(x_{\beta}^{1}, \ldots, x_{\beta}^{k}\right),
\end{aligned}
$$

which shows that lim sup epi $F_{\alpha} \subset$ epi $F_{\infty}$.

6. Operations involving functions and linear transformations. We continue with preservation results for convergence of convex functions, this time under operations involving linear transformations. Let $f$ be a convex function on $R^{n}, g$ a convex function on $R^{m}$, and $A$ a linear transformation from $R^{n}$ to $R^{m}$. The image of $f$ under $A$ is the convex function defined on $R^{m}$ by

$$
(A f)(y)=\inf \{f(x) \mid A x=y\}
$$

and the composition of $g$ and $A$ is the convex function defined on $R^{n}$ by

$$
(g A)(x)=g(A x) \text {. }
$$

The adjoint of $A$ is denoted by $A^{*}$. 
THEOREM 7. For each $\alpha=1, \ldots, \infty$ let $f_{\alpha}$ be a closed proper convex function on $R^{n}$ and let $A_{\alpha}$ be a linear transformation from $R^{n}$ to $R^{m}$. Assume that $f_{\alpha} \rightarrow f_{\infty}$ and $A_{\alpha} \rightarrow A_{\infty}$. Suppose that $N\left(A_{\infty}\right) \cap\left\{x \in R^{n} \mid\left(f_{\infty} 0^{+}\right)(x) \leqslant 0\right\}=\{0\}$. Then there is an $\bar{\alpha}$ such that $\alpha>\bar{\alpha}$ implies $A_{\alpha} f_{\alpha}$ is closed proper convex on $R^{m}$, and $A_{\alpha} f_{\alpha} \rightarrow A_{\infty} f_{\infty}$.

Proof. For each $\alpha=1, \ldots, \infty$ let $B_{\alpha}$ be the linear transformation from $R^{n+1}$ to $R^{m+1}$ defined by $B_{\alpha}(x, \mu)=\left(A_{\alpha} x, \mu\right)$. The definition of image of a convex function under a linear transformation implies that for each $\alpha$,

$$
B_{\alpha}\left(\text { epi } f_{\alpha}\right) \subset \operatorname{epi}\left(A_{\alpha} f_{\alpha}\right) \subset \operatorname{cl} B_{\alpha}\left(\text { epi } f_{\alpha}\right) \text {. }
$$

The hypothesis $N\left(A_{\infty}\right) \cap\left\{x \in R^{n} \mid\left(f_{\infty} 0^{+}\right)(x) \leqslant 0\right\}=\{0\}$ is equivalent to $N\left(B_{\infty}\right)$ $\cap 0^{+}\left(\right.$epi $\left.f_{\infty}\right)=\{0\}$. Hence, Theorem 3 yields an $\bar{\alpha}$ such that $\alpha>\bar{\alpha}$ implies $B_{\alpha}$ (epi $f_{\alpha}$ ) is closed, and $B_{\alpha}\left(\right.$ epi $\left.f_{\alpha}\right) \rightarrow B_{\infty}$ (epi $\left.f_{\infty}\right)$. Lemmas 4 and 5 imply that we may assume $N\left(B_{\alpha}\right) \cap 0^{+}\left(\right.$epi $\left.f_{\alpha}\right)=\{0\}$ for these $\alpha$, and so [13, Theorem 9.2] implies that for these $\alpha$ the function $A_{\alpha} f_{\alpha}$ is closed proper convex.

We remark that in Theorem 7 one also has, for each $\alpha>\bar{\alpha}$ and each fixed $y$ and $\mu$ satisfying $\left(A_{\alpha} f_{\alpha}\right)(y) \leqslant \mu<+\infty$, that the set $\left\{x \in R^{n} \mid A_{\alpha} x=y, f_{\alpha}(x) \leqslant \mu\right\}$ is actually compact convex. This can be shown as an exercise by means of recession theory [13] since, as was shown in the proof above, the condition $N\left(A_{\alpha}\right) \cap\{x \in$ $\left.R^{n} \mid\left(f_{\alpha} 0^{+}\right)(x) \leqslant 0\right\}=\{0\}$ holds for such $\alpha$.

The condition needed for Theorem 7 will now be dualized in preparation for Theorem 8.

LEMMA 7. Let $f$ be a closed proper convex function on $R^{n}$, and let $A$ be a linear transformation from $R^{m}$ to $R^{n}$. Then the following three conditions,

$$
\begin{gathered}
N\left(A^{*}\right) \cap\left\{x^{*} \in R^{n} \mid\left(f^{*} 0^{+}\right)\left(x^{*}\right) \leqslant 0\right\}=\{0\}, \\
R(A)^{\perp} \cap\left\{x^{*} \in R^{n} \mid \delta^{*}\left(x^{*} \mid \operatorname{dom} f\right) \leqslant 0\right\}=\{0\}, \\
0 \in \operatorname{int}(R(A)-\operatorname{dom} f),
\end{gathered}
$$

are pairwise equivalent, and are implied by

$$
\varnothing \neq R(A) \cap \operatorname{int}(\operatorname{dom} f) .
$$

Proof. It is standard that $R(A)^{\perp}=N\left(A^{*}\right)$, and [13, Theorem 13.3] yields $\delta^{*}(\cdot \mid \operatorname{dom} f)=f^{*} 0^{+}$. The equivalence of $(6.1)$ and (6.2) is now immediate. To see the equivalence between (6.2) and (6.3), observe that (6.2) is equivalent to

$$
\delta^{*}\left(x^{*} \mid R(A)\right)+\delta^{*}\left(x^{*} \mid \operatorname{dom} f\right)>0, \quad \forall x^{*} \neq 0,
$$

since $\delta\left(\cdot \mid R(A)^{\perp}\right)$ and $\delta^{*}(\cdot \mid R(A))$ are equal. This condition can be written as $\phi 0^{+}\left(x^{*}\right)>0, \forall x^{*} \neq 0$, for $\phi$ the closed proper convex function

$$
\phi=\delta^{*}(\cdot \mid R(A))+\delta^{*}(\cdot \mid-\operatorname{dom} f)=\delta^{*}(\cdot \mid R(A)-\operatorname{dom} f) .
$$

By [13, Corollary $13.3 .4(\mathrm{c})]$ the latter condition is equivalent to the condition $0 \in \operatorname{int}\left(\operatorname{dom} \phi^{*}\right)$. Since $\phi^{*}=\delta(\cdot \mid R(A)-\operatorname{dom} f)$, this is the same as (6.3). Finally, since

$$
R(A)-\operatorname{int}(\operatorname{dom} f) \subset \operatorname{int}(R(A)-\operatorname{dom} f),
$$

it is clear that (6.4) implies (6.3). 
THEOREM 8. For each $\alpha=1, \ldots, \infty$ let $f_{\alpha}$ be a closed proper convex function on $R^{n}$ and let $A_{\alpha}$ be a linear transformation from $R^{m}$ to $R^{n}$. Assume that $f_{\alpha} \rightarrow f_{\infty}$ and $A_{\alpha} \rightarrow A_{\infty}$. Suppose that $0 \in \operatorname{int}\left(R\left(A_{\infty}\right)-\operatorname{dom} f_{\infty}\right)$. Then there is an $\bar{\alpha}$ such that $\alpha>\bar{\alpha}$ implies $f_{\alpha} A_{\alpha}$ is closed proper convex on $R^{m}$, and $f_{\alpha} A_{\alpha} \rightarrow f_{\infty} A_{\infty}$.

Proof. Lemma 7 implies that $N\left(A_{\infty}^{*}\right) \cap\left\{x^{*} \in R^{n} \mid\left(f_{\infty}^{*} 0^{+}\right)\left(x^{*}\right) \leqslant 0\right\}=\{0\}$. Theorem 1 implies $f_{\alpha}^{*} \rightarrow f_{\infty}^{*}$, and clearly $A_{\alpha}^{*} \rightarrow A_{\infty}^{*}$. From Theorem 7 we have an $\bar{\alpha}$ such that $\alpha>\bar{\alpha}$ implies $A_{\alpha}^{*} f_{\alpha}^{*}$ is closed proper convex, and $A_{\alpha}^{*} f_{\alpha}^{*} \rightarrow A_{\infty}^{*} f_{\infty}^{*}$. Theorem 1 now yields $\left(A_{\alpha}^{*} f_{\alpha}^{*}\right)^{*} \rightarrow\left(A_{\infty}^{*} f_{\infty}^{*}\right)^{*}$. Since $\left(A_{\alpha}^{*} f_{\alpha}^{*}\right)^{*}=f_{\alpha}^{* *} A_{\alpha}^{* *}=f_{\alpha} A_{\alpha}$ for each $\alpha$, we obtain $f_{\alpha} A_{\alpha} \rightarrow f_{\infty} A_{\infty}$, where $f_{\alpha} A_{\alpha}$ is closed and proper for all $\alpha>\bar{\alpha}$ (see [13, Theorems 16.3, 12.2]).

7. Addition of subdifferentials. We now recast Theorem 5 in terms of subdifferentials, a special class of maximal monotone operators. Similar results for other classes of maximal monotone operators may be found in [3, Chapter 5] and [2].

THEOREM 9. For each $\alpha=1, \ldots, \infty$ let $M_{\alpha}$ and $L_{\alpha}$ be subdifferentials of closed proper convex functions on $R^{n}$. Assume that $G\left(M_{\alpha}\right) \rightarrow G\left(M_{\infty}\right), G\left(L_{\alpha}\right) \rightarrow G\left(L_{\infty}\right)$, and $0 \in \operatorname{int}\left(\operatorname{dom} M_{\infty}-\operatorname{dom} L_{\infty}\right)$. Then there is an $\bar{\alpha}$ such that $\alpha>\bar{\alpha}$ implies $\left(M_{\alpha}+\right.$ $\left.L_{\alpha}\right)$ is the subdifferential of a closed proper convex function on $R^{n}$, and $G\left(M_{\alpha}+L_{\alpha}\right)$ $\rightarrow G\left(M_{\infty}+L_{\infty}\right)$.

PROOF. For each $\alpha$ we have a closed proper convex function $f_{\alpha}$ on $R^{n}$ such that $M_{\alpha}=\partial f_{\alpha}$. As $G\left(M_{\infty}\right)$ is contained in lim inf $G\left(M_{\alpha}\right)$, there exist $\left(x_{\infty}, y_{\infty}\right) \in G\left(M_{\infty}\right)$ and a sequence $\left(\left(x_{\alpha}, y_{\alpha}\right)\right)$ such that $\left(x_{\alpha}, y_{\alpha}\right) \in G\left(M_{\alpha}\right)$ and $\left(x_{\alpha}, y_{\alpha}\right) \rightarrow\left(x_{\infty}, y_{\infty}\right)$. For each $\alpha=1, \ldots, \infty$ set $c_{\alpha}=f_{\alpha}\left(x_{\alpha}\right) \in R$, and define functions $\phi_{\alpha}$ on $R^{n}$ by

$$
\phi_{\alpha}(x)=f_{\alpha}(x)-c_{\alpha} \text {. }
$$

Then $M_{\alpha}=\partial \phi_{\alpha}$ and $\phi_{\alpha}\left(x_{\alpha}\right)=0$ for all $\alpha$, and $x_{\alpha} \rightarrow x_{\infty}$, so Theorem 2 yields $\phi_{\alpha} \rightarrow \phi_{\infty}$. In the same manner, we can find closed proper convex functions $\psi_{\alpha}$ such that $L_{\alpha}=\partial \psi_{\alpha}$ and $\psi_{\alpha} \rightarrow \psi_{\infty}$. Since the domain of a closed proper convex function contains the domain of its subdifferential, the hypothesis

$$
0 \in \operatorname{int}\left(\operatorname{dom} M_{\infty}-\operatorname{dom} L_{\infty}\right)
$$

implies $0 \in \operatorname{int}\left(\operatorname{dom} \phi_{\infty}-\operatorname{dom} \psi_{\infty}\right)$. It follows from Theorem 5 that there is an $\bar{\alpha}$ such that $\alpha>\bar{\alpha}$ implies $\phi_{\alpha}+\psi_{\alpha}$ is closed proper convex, and $\left(\phi_{\alpha}+\psi_{\alpha}\right) \rightarrow$ $\left(\phi_{\infty}+\psi_{\infty}\right)$. Theorem 2 yields $G\left(\partial\left(\phi_{\alpha}+\psi_{\alpha}\right)\right) \rightarrow G\left(\partial\left(\phi_{\infty}+\psi_{\infty}\right)\right)$. It is easy to check that

$$
\begin{aligned}
\left(\operatorname{dom} \phi_{\infty}-\operatorname{dom} \psi_{\infty}\right) & \subset\left(\lim \inf \left(\operatorname{dom} \phi_{\alpha}\right)-\lim \inf \left(\operatorname{dom} \psi_{\alpha}\right)\right) \\
& \subset \lim \inf \left(\operatorname{dom} \phi_{\alpha}-\operatorname{dom} \psi_{\alpha}\right),
\end{aligned}
$$

so [9, Lemma 4] implies we may assume $0 \in \operatorname{int}\left(\operatorname{dom} \phi_{\alpha}-\operatorname{dom} \psi_{\alpha}\right.$ ) for all $\alpha>\bar{\alpha}$. For these $\alpha$ we have from [14, Theorem 20(c)] that $\partial\left(\phi_{\alpha}+\psi_{\alpha}\right)=\partial \phi_{\alpha}+\partial \psi_{\alpha}$. We now have shown that $G\left(M_{\alpha}+L_{\alpha}\right) \rightarrow G\left(M_{\infty}+L_{\infty}\right)$.

8. Generalizations to more than two (sequences of) functions. Whenever possible, the following results are labelled with primes to conform to the labels of their previous special cases. 
Corollary $3 \mathrm{~A}^{\prime}$. For each $\alpha=1, \ldots, \infty$ and $i=1, \ldots, k$ let $C_{\alpha}^{i}$ be a nonempty convex subset of $R^{n}$. Assume that $C_{\alpha}^{i} \rightarrow C_{\infty}^{i}$ for each $i$. Suppose that

$$
\left.\begin{array}{l}
z_{1}+\cdots+z_{k}=0 \\
z_{i} \in 0^{+} C_{\infty}^{i} \forall i
\end{array}\right\} \Rightarrow z_{i}=0 \quad \forall i
$$

Then $\left(C_{\alpha}^{1}+\cdots+C_{\alpha}^{k}\right) \rightarrow\left(C_{\infty}^{1}+\cdots+C_{\infty}^{k}\right)$. If for each $\alpha$ and $i$ the sets $C_{\alpha}^{i}$ are also closed, then there is an $\bar{\alpha}$ such that $\alpha>\bar{\alpha}$ implies $\left(C_{\alpha}^{1}+\cdots+C_{\alpha}^{k}\right)$ is closed.

PROOF. An obvious extension of Corollary 3A.

LEMMA $6^{\prime}$. For each $i=1, \ldots, k$ let $f^{i}$ be a closed proper convex function on $R^{n}$, and let the conjugate of $f^{i}$ be written as $g^{i}$. Then the following three conditions

$$
\begin{aligned}
& \left.\begin{array}{l}
\left(y_{1}, \eta^{1}\right)+\cdots+\left(y_{k}, \eta^{k}\right)=(0,0) \\
\left(y_{i}, \eta^{i}\right) \in 0^{+}\left(\text {epi } g^{i}\right) \quad \forall i
\end{array}\right\} \Rightarrow\left(y_{i}, \eta^{i}\right)=(0,0) \quad \forall i, \\
& \left.\begin{array}{l}
y_{1}+\cdots+y_{k}=0 \\
\left(g^{1} 0^{+}\right)\left(y_{1}\right)+\cdots+\left(g^{k} 0^{+}\right)\left(y_{k}\right) \leqslant 0
\end{array}\right\} \Rightarrow y_{i}=0 \quad \forall i, \\
& 0 \in \operatorname{int}\left\{\left(x_{1}, \ldots, x_{k}\right) \mid \varnothing \neq \bigcap_{i=1}^{k}\left(x_{i}+\operatorname{dom} f^{i}\right)\right\}
\end{aligned}
$$

are pairwise equivalent, and are implied by

$$
\varnothing \neq \operatorname{dom} f^{1} \cap \operatorname{int}\left(\operatorname{dom} f^{2}\right) \cap \cdots \cap \operatorname{int}\left(\operatorname{dom} f^{k}\right) .
$$

Proof. Clearly, (8.2) implies (8.1). To prove the converse, consider any $k$-tuple $\left(y_{1}, \ldots, y_{k}\right)$ satisfying the premise of (8.2). Define $\eta^{i}=\left(g^{i} 0^{+}\right)\left(y_{i}\right)$ for $i=$ $2, \ldots, k$ and $\eta^{1}=-\left(\eta^{2}+\cdots+\eta^{k}\right)$. Since these quantities satisfy $\left(y_{i}, \eta^{i}\right) \in$ epi $\left(g^{i} 0^{+}\right)$for each $i$, (8.1) applies to yield $y_{i}=0$ for each $i$. Thus, (8.1) implies (8.2). Next, we consider a special case of the equivalence between (6.1) and (6.3) in Lemma 7. Specifically, consider

$$
\begin{gathered}
A(x)=(x, \ldots, x) \quad(k \text { copies }) \\
F\left(x_{1}, \ldots, x_{k}\right)=f^{1}\left(x_{1}\right)+\cdots+f^{k}\left(x_{k}\right)
\end{gathered}
$$

Then

$$
\begin{aligned}
A^{*}\left(y_{1}, \ldots, y_{k}\right) & =y_{1}+\cdots+y_{k}, \\
F^{*}\left(y_{1}, \ldots, y_{k}\right) & =g^{1}\left(y_{1}\right)+\cdots+g^{k}\left(y_{k}\right) .
\end{aligned}
$$

Also,

$$
\begin{gathered}
\operatorname{dom} F=\operatorname{dom} f^{1} \times \cdots \times \operatorname{dom} f^{k}, \\
\left(F^{*} 0^{+}\right)\left(y_{1}, \ldots, y_{k}\right)=\left(g^{1} 0^{+}\right)\left(y_{1}\right)+\cdots+\left(g^{k} 0^{+}\right)\left(y_{k}\right) .
\end{gathered}
$$

From these formulas and Lemma 7, the equivalence between (8.2) and (8.3) is clear. Finally, assume that (8.4) holds. Then there exists an $x$ satisfying

$$
x \in \operatorname{dom} f^{1} \cap \operatorname{int}\left(\operatorname{dom} f^{2}\right) \cap \cdots \cap \operatorname{int}\left(\operatorname{dom} f^{k}\right) .
$$

So for each $i=2, \ldots, k$ there exists an $\varepsilon^{i}>0$ such that $x+2 \varepsilon^{i} B \subset \operatorname{dom} f^{i}$, where $B$ denotes the unit ball in $R^{n}$. Put $\varepsilon^{1}=\min \left\{\varepsilon^{2}, \ldots, \varepsilon^{k}\right\}$. Consider any 
$\left(x_{1}, \ldots, x_{k}\right) \in \varepsilon^{1} B \times \cdots \times \varepsilon^{k} B$. Then $x_{1}+x \in x_{1}+\operatorname{dom} f^{1}$, while for each $i=$ $2, \ldots, k$ we have

$$
x_{1}+x=x_{i}+x+\left(x_{1}-x_{i}\right) \in x_{i}+\operatorname{dom} f^{i},
$$

because

$$
x_{1}-x_{i} \in \varepsilon^{1} B+\varepsilon^{i} B \subset 2 \varepsilon^{i} B .
$$

This shows that

$$
\varnothing \neq \bigcap_{i=1}^{k}\left(x_{i}+\operatorname{dom} f^{i}\right)
$$

Hence,

$$
\varepsilon^{1} B \times \cdots \times \varepsilon^{k} B \subset\left\{\left(x_{1}, \ldots, x_{k}\right) \mid \varnothing \neq \bigcap_{i=1}^{k}\left(x_{i}+\operatorname{dom} f^{i}\right)\right\},
$$

which establishes (8.3).

Lemma 8. For each $\alpha=1, \ldots, \infty$ and $i=1, \ldots, k$ let $f_{\alpha}^{i}$ be a closed proper convex function on $R^{n}$. Assume that $f_{\alpha}^{i} \rightarrow f_{\infty}^{i}$ for each $i$. Suppose that

$$
0 \in \operatorname{int}\left\{\left(x_{1}, \ldots, x_{k}\right) \mid \varnothing \neq \bigcap_{i=1}^{k}\left(x_{i}+\operatorname{dom} f_{\infty}^{i}\right)\right\} .
$$

Then there is an $\bar{\alpha}$ such that $\alpha>\bar{\alpha}$ implies

$$
0 \in \operatorname{int}\left\{\left(x_{1}, \ldots, x_{k}\right) \mid \varnothing \neq \bigcap_{i=1}^{k}\left(x_{i}+\operatorname{dom} f_{\alpha}^{i}\right)\right\} .
$$

Proof. From the proof of Lemma $6^{\prime}$ we know the conditions under discussion are of the form $0 \in \operatorname{int}\left(R(A)-\operatorname{dom} F_{\alpha}\right)$, where

$$
\begin{aligned}
A(x) & =(x, \ldots, x), \\
F_{\alpha}\left(x_{1}, \ldots, x_{k}\right) & =f_{\alpha}^{1}\left(x_{1}\right)+\cdots+f_{\alpha}^{k}\left(x_{k}\right) .
\end{aligned}
$$

By Lemma 7 this condition is equivalent to

$$
N\left(A^{*}\right) \cap\left\{\left(y_{1}, \ldots, y_{k}\right) \mid G_{\alpha} 0^{+}\left(y_{1}, \ldots, y_{k}\right) \leqslant 0\right\}=\{0\},
$$

where

$$
\begin{aligned}
& A^{*}\left(y_{1}, \ldots, y_{k}\right)=y_{1}+\cdots+y_{k}, \\
& G_{\alpha}\left(y_{1}, \ldots, y_{k}\right)=g_{\alpha}^{1}\left(y_{1}\right)+\cdots+g_{\alpha}^{k}\left(y_{k}\right) .
\end{aligned}
$$

(Here, as earlier, we write $g_{\alpha}^{i}=\left(f_{\alpha}^{i}\right)^{*}$.) Now Theorem 1 yields $g_{\alpha}^{i} \rightarrow g_{\infty}^{i}$ for each $i$, so Theorem 6 yields $G_{\alpha} \rightarrow G_{\infty}$. By Lemma 4 this yields

$$
\text { lim sup epi }\left(G_{\alpha} 0^{+}\right) \subset \operatorname{epi}\left(G_{\infty} 0^{+}\right) \text {, }
$$

which easily implies that the set

$$
\lim \sup \left\{\left(y_{1}, \ldots, y_{k}\right) \mid G_{\alpha} 0^{+}\left(y_{1}, \ldots, y_{k}\right)<0\right\}
$$


is contained in $\left\{\left(y_{1}, \ldots, y_{k}\right) \mid G_{\infty} 0^{+}\left(y_{1}, \ldots, y_{k}\right) \leqslant 0\right\}$. Since our hypothesis is that $N\left(A^{*}\right) \cap\left\{\left(y_{1}, \ldots, y_{k}\right) \mid G_{\infty} 0^{+}\left(y_{1}, \ldots, y_{k}\right) \leqslant 0\right\}=\{0\}$, Lemma 5 yields an $\bar{\alpha}$ such that

$$
N\left(A^{*}\right) \cap\left\{\left(y_{1}, \ldots, y_{k}\right) \mid G_{\alpha} 0^{+}\left(y_{1}, \ldots, y_{k}\right) \leqslant 0\right\}=\{0\}
$$

holds whenever $\alpha>\bar{\alpha}$. The proof is concluded by retracing our steps (via the definitions of $A^{*}, G_{\alpha}, A, F_{\alpha}$ together with Lemmas 7 and $6^{\prime}$ ) to rewrite the latter condition in terms of the $f_{\alpha}^{i}$.

TheOREM 4'. For each $\alpha=1, \ldots, \infty$ and $i=1, \ldots, k$ let $g_{\alpha}^{i}$ be a closed proper convex function on $R^{n}$. Assume that $g_{\alpha}^{i} \rightarrow g_{\infty}^{i}$ for each $i$. Suppose that

$$
\left.\begin{array}{l}
\left(y_{1}, \eta^{1}\right)+\cdots+\left(y_{k}, \eta^{k}\right)=(0,0) \\
\left(y_{i}, \eta^{i}\right) \in 0^{+}\left(\text {epi } g_{\infty}^{i}\right) \quad \forall i
\end{array}\right\} \Rightarrow\left(y_{i}, \eta^{i}\right)=(0,0) \quad \forall i .
$$

Then there is an $\bar{\alpha}$ such that $\alpha>\bar{\alpha}$ implies $\left(g_{\alpha}^{1} \square \cdots \square g_{\alpha}^{k}\right)$ is closed proper convex, and $\left(g_{\alpha}^{1} \square \cdots \square g_{\alpha}^{k}\right) \rightarrow\left(g_{\infty}^{1} \square \cdots \square g_{\infty}^{1}\right)$.

Proof. From the definition of infimal convolution, for each $\alpha$ we have

$$
\left(\text { epi } g_{\alpha}^{1}+\cdots+\text { epi } g_{\alpha}^{k}\right) \subset \operatorname{epi}\left(g_{\alpha}^{1} \square \cdots \square g_{\alpha}^{k}\right) \subset \operatorname{cl}\left(\text { epi } g_{\alpha}^{1}+\cdots+\text { epi } g_{\alpha}^{k}\right) \text {. }
$$

By Corollary $3 \mathrm{~A}^{\prime}$, there is an $\bar{\alpha}$ such that $\alpha>\bar{\alpha}$ implies (epi $g_{\alpha}^{1}+\cdots+$ epi $g_{\alpha}^{k}$ ) is closed, and (epi $g_{\alpha}^{1}+\cdots+$ epi $\left.g_{\alpha}^{k}\right) \rightarrow\left(\right.$ epi $g_{\infty}^{1}+\cdots+$ epi $g_{\infty}^{k}$ ). This yields $\left(g_{\alpha}^{1} \square \cdots \square g_{\alpha}^{k}\right) \rightarrow\left(g_{\infty}^{1} \square \cdots \square g_{\infty}^{k}\right)$. By (8.5) together with Lemmas $6^{\prime}$ and 8, we may assume that the condition

$$
\left.\begin{array}{l}
\left(g_{\alpha}^{1} 0^{+}\right)\left(y_{1}\right)+\cdots+\left(g_{\alpha}^{k} 0^{+}\right)\left(y_{k}\right) \leqslant 0 \\
y_{1}+\cdots+y_{k}=0
\end{array}\right\} \Rightarrow y_{i}=0 \quad \forall i
$$

holds for all $\alpha>\bar{\alpha}$. Hence, for these $\alpha$, [13, Corollary 9.2.1] implies that $\left(g_{\alpha}^{1} \square \cdots \square g_{\alpha}^{k}\right)$ is closed proper convex.

We remark that in Theorem $4^{\prime}$, for all $\alpha>\bar{\alpha}$ and all $y$ and $\mu$ satisfying $\left(g_{\alpha}^{1} \square \cdots \square g_{\alpha}^{k}\right)(y) \leqslant \mu<+\infty$, the set

$$
\left\{\left(y_{1}, \ldots, y_{k}\right) \mid y=y_{1}+\cdots+y_{k}, g_{\alpha}^{1}\left(y_{1}\right)+\cdots+g_{\alpha}^{k}\left(y_{k}\right) \leqslant \mu\right\}
$$

is actually compact convex. This can be seen as a special case of the analogous remark following Theorem 7.

THEOREM $5^{\prime}$. For each $\alpha=1, \ldots, \infty$ and $i=1, \ldots, k$ let $f_{\alpha}^{i}$ be a closed proper convex function on $R^{n}$. Assume that $f_{\alpha}^{i} \rightarrow f_{\infty}^{i}$ for each $i$. Suppose that

$$
0 \in \operatorname{int}\left\{\left(x_{1}, \ldots, x_{k}\right) \mid \varnothing \neq \bigcap_{i=1}^{k}\left(x_{i}+\operatorname{dom} f_{\infty}^{i}\right)\right\} .
$$

Then there is an $\bar{\alpha}$ such that $\alpha>\bar{\alpha}$ implies $\left(f_{\alpha}^{1}+\cdots+f_{\alpha}^{k}\right)$ is closed proper convex, and $\left(f_{\alpha}^{1}+\cdots+f_{\alpha}^{k}\right) \rightarrow\left(f_{\infty}^{1}+\cdots+f_{\infty}^{k}\right)$.

Proof. By Theorem $1, g_{\alpha}^{i} \rightarrow g_{\infty}^{i}$ for each $i$, where we write $g_{\alpha}^{i}=\left(f_{\alpha}^{i}\right)^{*}$. Hence, by (8.6) together with Lemma $6^{\prime}$ and Theorem $4^{\prime}$, there exists an $\bar{\alpha}$ such that $\alpha>\bar{\alpha}$ implies $\left(g_{\alpha}^{1} \square \cdots \square g_{\alpha}^{k}\right)$ is closed proper convex, and $\left(g_{\alpha}^{1} \square \cdots \square g_{\alpha}^{k}\right)$ $\rightarrow\left(g_{\infty}^{1} \square \cdots \square g_{\infty}^{k}\right)$. Since $\left(g_{\alpha}^{1} \square \cdots \square g_{\alpha}^{k}\right)=\left(f_{\alpha}^{1}+\cdots+f_{\alpha}^{k}\right)^{*}$ and 
$\left(f_{\alpha}^{1}+\cdots+f_{\alpha}^{k}\right)$ is closed convex, it follows that for all $\alpha>\bar{\alpha}$ the function $\left(f_{\alpha}^{1}+\cdots+f_{\alpha}^{k}\right)$ is proper (see [13, Theorems 16.4, 9.3, 12.2]). Finally, Theorem 1 yields $\left(f_{\alpha}^{1}+\cdots+f_{\alpha}^{k}\right) \rightarrow\left(f_{\infty}^{1}+\cdots+f_{\infty}^{k}\right)$.

THEOREM $9^{\prime}$. For each $\alpha=1, \ldots, \infty$ and $i=1, \ldots, k$ let $M_{\alpha}^{i}$ be a subdifferential of a closed proper convex function on $R^{n}$. Assume that $G\left(M_{\alpha}^{i}\right) \rightarrow G\left(M_{\infty}^{i}\right)$ for each $i$. Suppose that

$$
0 \in \operatorname{int}\left\{\left(x_{1}, \ldots, x_{k}\right) \mid \varnothing \neq \bigcap_{i=1}^{k}\left(x_{i}+D\left(M_{\infty}^{i}\right)\right)\right\} .
$$

Then there is an $\bar{\alpha}$ such that $\alpha>\bar{\alpha}$ implies $\left(M_{\alpha}^{1}+\cdots+M_{\alpha}^{k}\right)$ is the subdifferential of a closed proper convex function on $R^{n}$, and

$$
G\left(M_{\alpha}^{1}+\cdots+M_{\alpha}^{k}\right) \rightarrow G\left(M_{\infty}^{1}+\cdots+M_{\infty}^{k}\right) .
$$

Proof. We begin just as in the proof of Theorem 9, obtaining closed proper convex functions $\varphi_{\alpha}^{i}$ such that $M_{\alpha}^{i}=\partial \varphi_{\alpha}^{i}$, where $\varphi_{\alpha}^{i} \rightarrow \varphi_{\infty}^{i}$ for each $i$. Next, since $\operatorname{dom} \varphi_{\alpha}^{i} \supset D\left(M_{\alpha}^{i}\right),(8.7)$ yields

$$
0 \in \operatorname{int}\left\{\left(x_{1}, \ldots, x_{k}\right) \mid \varnothing \neq \bigcap_{i=1}^{k}\left(x_{i}+\operatorname{dom} \varphi_{\infty}^{i}\right)\right\} .
$$

Therefore, Theorem $5^{\prime}$ yields an $\bar{\alpha}$ such that $\alpha>\bar{\alpha}$ implies $\varphi_{\alpha}^{1}+\cdots+\varphi_{\alpha}^{k}$ is closed proper convex, and $\left(\varphi_{\alpha}^{1}+\cdots+\varphi_{\alpha}^{k}\right) \rightarrow\left(\varphi_{\infty}^{1}+\cdots+\varphi_{\infty}^{k}\right)$. By Theorem 2 , this implies that $G\left(\partial\left(\varphi_{\alpha}^{1}+\cdots+\varphi_{\alpha}^{k}\right)\right) \rightarrow G\left(\partial\left(\varphi_{\infty}^{1}+\cdots+\varphi_{\infty}^{k}\right)\right)$. Finally, by Lemma 8 and (8.8), we can assume that the condition

$$
0 \in \operatorname{int}\left\{\left(x_{1}, \ldots, x_{k}\right) \mid \varnothing \neq \bigcap_{i=1}^{k}\left(x_{i}+\operatorname{dom} \varphi_{\alpha}^{i}\right)\right\}
$$

holds for all $\alpha>\bar{\alpha}$. Therefore, for those $\alpha$, [14, Theorem 20(c)] implies that $\partial\left(\varphi_{\alpha}^{1}+\cdots+\varphi_{\alpha}^{k}\right)=\partial \varphi_{\alpha}^{1}+\cdots+\partial \varphi_{\alpha}^{k}$. This completes the proof.

We remark, concerning the technical conditions appearing in Theorem $9^{\prime}$ and its proof, that it is not hard to show that the set appearing in (8.9) and the set

$$
\operatorname{int}\left\{\left(x_{1}, \ldots, x_{k}\right) \mid \varnothing \neq \bigcap_{i=1}^{k}\left(x_{i}+D\left(M_{\alpha}^{i}\right)\right)\right\}
$$

actually coincide, where $M_{\alpha}^{i}=\partial \varphi_{\alpha}^{i}$.

\section{REFERENCES}

1. H. Attouch, Familles d'opérateurs maximaux monotones et mesurabilité, Ann. Mat. Pura Appl. 120 (1979), 35-111.

2. H. Attouch and Y. Konishi, Convergence d'opérateurs maximaux monotones et inéquations variationelles, C. R. Acad. Sci. Paris Sér. A-B 282 (1976), A467-A469.

3. R. C. Bergstrom, Optimization, convergence, and duality, Thesis, Univ. of Illinois at UrbanaChampaign, 1980.

4. R. C. Bergstrom and L. McLinden, Convergent sequences of dual convex programs (submitted).

5. H. Brézis, Opérateurs maximaux monotones et semi-groupes de contractions dans les espaces de Hilbert, North-Holland, Amsterdam, 1973.

6. J. L. Joly, Une famille de topologies et de convergences sur l'ensemble des fonctionelles convexes, Thesis, Univ. Scientifique et Médicale de Grenoble, Grenoble, 1970. 
7. , Une famille de topologies sur l'ensembles des fonctions convexes pour lesquelles la polarité est bicontinue, J. Math. Pures Appl. 52 (1973), 421-441.

8. M. Matzeu, Su un tipo de continuata' dell'operatore subdifferenziale, Boll. Un. Mat. Ital. B(5) 14 (1977), 480-490.

9. L. McLinden, Successive approximation and linear stability involving convergent sequences of optimization problems (submitted).

10. Convergent sequences of minimax problems and saddle functions (in preparation).

11. U. Mosco, Convergence of convex sets and of solutions of variational inequalities, Adv. in Math. 3 (1969), 510-585.

12. __ On the continuity of the Young-Fenchel transform, J. Math. Anal. Appl. 35 (1971), 518-535.

13. R. T. Rockafellar, Convex analysis, Princeton Univ. Press, Princeton, N. J., 1970.

14. __ Conjugate duality and optimization, Regional Conf. Ser. Appl. Math., no. 16, SIAM, Philadelphia, Pa., 1974.

15. G. Salinetti and R. J.-B. Wets, On the relations between two types of convergence for convex functions, J. Math. Anal. Appl. 60 (1977), 211-226.

16. , On the convergence of sequences of convex sets in finite dimensions, SIAM Rev. 21 (1979), $18-33$.

17. D. W. Walkup and R. J.-B. Wets, Continuity of some convex-cone-valued mappings, Proc. Amer. Math. Soc. 18 (1967), 229-235.

18. R. J.-B. Wets, Convergence of convex functions, variational inequalities, and convex optimization problems, Variational Inequalities and Complementarity Problems (R. W. Cottle, F. Giannessi and J.-L. Lions, eds.), Wiley, London, 1980, pp. 375-403.

19. R. A. Wijsman, Convergence of sequences of convex sets, cones, and functions, Bull. Amer. Math. Soc. 70 (1964), 186-188.

20. Convergence of sequences of convex sets, cones, and functions. II, Trans. Amer. Math. Soc. 123 (1966), 32-45.

Department of Mathematics, University of IllinoIs, Urbana, Illinois 61801

Department of Mathematics, University of the Pacific, Stockton, California 95211 